E-Review Dossier 6-2018

Bologna (BraDypUS)

I molti territori della Repubblica fascista. Amministrazione e società nella RSI a cura di Roberto Parisini, Roberta Mira e Toni Rovatti

\section{Amministrazione e società nella Repubblica sociale italiana. Una proposta interpretativa}

ISSN: 2282-4979

DOI: $10.12977 /$ ere-

view259

Verificare l'effettiva consistenza della Rsi e il suo significato come ponte tra fascismo e Italia democratica non può non passare attraverso l'analisi del funzionamento della sua struttura amministrativa. È questo infatti un canale fondamentale che Salò attiva per avere consenso/accettazione da parte di diversi strati della società italiana, soprattutto all'interno della zona grigia. Ma viste le indiscutibili impotenze del centro, importante è saldare questa analisi alle caratteristiche che, negli anni Trenta, assume l'impianto dello stato amministrativo e corporativo incontrando le specificità delle diverse periferie. Caratteristiche e specificità che vengono poi, in ogni territorio, accentuate o ridefinite dalle frammentazioni direttamente connesse agli eventi bellici.

Verify the actual consistency of the Italian Social Republic and its meaning as a connection between Fascism and Democratic Italy: this happens through the analysis of the functioning of its administrative structure. In fact, this is a fundamental channel which Salo activated to be accepted by different sections of the Italian society, above all in the grey area. Given the undeniable weakness of the central government, it is important to connect this analysis to the characteristics of the administrative and corporate State in the Thirties, taking the peculiarities of the different peripheral territories into account. Characteristics and peculiarities which are then emphasized or redefined in each territory according to the disruptions directly linked to war.

Nel corso degli anni Novanta, la Repubblica sociale italiana è stata oggetto di un'attenta rivisitazione, in parte anticipata, a metà del decennio precedente, da un importante convegno organizzato dalla Fondazione Micheletti [Poggio 1986]. Una maggiore complessità e articolazione degli studi sulla Resistenza ha aperto la via a quella fortunata stagione che ha finalmente immesso la Repubblica fascista nelle linee della storia nazionale. Sono emersi angoli d'osservazione diversi, 
che hanno sensibilmente ridimensionato alcuni stereotipi interpretativi fino allora consolidati. Questi andavano dalla totale inconsistenza politica e amministrativa dell'ultimo stato fascista, inevitabilmente condannato perciò a una servile efferatezza; fino alla mitologia reducistica della "repubblica necessaria", dove l'azione moderata e protettiva di Mussolini e dei suoi funzionari sarebbe stata funestata da fascisti fanatici e intransigenti, che avrebbero reso nella sostanza ingovernabile il nuovo stato [Della Valle 1978].

Ora, calata nel contesto della guerra civile e approfondito quello della complessa relazione con l'alleato-occupante [Pavone 1991; Klinkhammer 1993], si faceva strada una maggiore attenzione all'effettiva consistenza della Rsi, a una sua specifica periodizzazione interna. Si usciva da contrapposizioni troppo secche (moderati/intransigenti, stato/partito, ecc.) e da fallimenti troppo scontati [Legnani 1986]; si davano articolazione e profondità alle motivazioni di chi vi aderì, così come alle parole d'ordine volte a suscitare consenso; e anche alle violenze che, allo stesso modo, attraversarono la Rsi per tutto l'arco della sua breve vita [Gagliani 1999; Ganapini 1999]. Tutti temi che, nuovi o rinnovati che fossero, guadagnavano poi senso complessivo ponendosi il problema dell'interpretazione del biennio 19431945 come ponte tra il regime fascista e la repubblica del dopoguerra'.

Tra essi spiccava la questione della zona grigia, ossia di tutta quella parte della società italiana che non entrò in nessuno dei campi in lotta, ma al più rifluì dall'uno all'altro, a seconda dei momenti e dei contesti. Accanto a questa, veniva perciò profilata la necessità di ricostruire quei canali (etico-politici o puramente praticoamministrativi) attraverso i quali la Rsi si era rivolta a diversi gruppi di italiani, ottenendone forme di consenso o anche, certamente più spesso, di adeguamento/ accettazione; comunque forme attraverso le quali pezzi consistenti della società furono traghettati fin dentro l'Italia democratica [Pavone 1995]. Si trattava di una questione ampia che, pur dotata di una propria fisionomia segnata dal prepotente bisogno di sopravvivere, tendeva a rivelarsi difficile e sfuggente quanto altre 
mai'. Essa, infatti, finiva spesso per lambire un po' tutte quelle problematiche di cui pure si discusse più a lungo: dall'identificazione delle molte e variabili gradazioni del collaborazionismo e dell'opposizione, all'effettivo valore fondativo della Resistenza e dell'antifascismo [Rusconi 1995; Scoppola 1995; De Felice 1997; Pavone 1998].

Tuttavia, sollecitati dagli eventi pubblici e dalle guerre degli anni Novanta che hanno spinto in primo piano lo sforzo di rielaborare una memoria collettiva ${ }^{3}$, gli studi degli anni successivi hanno ulteriormente sviluppato alcuni dei temi citati, in particolare quelli legati alla violenza [Osti Guerrazzi 2012; Rovatti 2011 e 2014] mentre altri, non meno cruciali, sono caduti piuttosto in ombra. È stato questo il destino occorso appunto alla zona grigia, anche se è dietro di essa che si staglia in buona misura il problema di tracciare un serio bilancio delle concrete capacità di governo, delle strategie politico-amministrative non sempre infruttuose che il nuovo stato fascista attivò - soprattutto a partire dal profilarsi del consolidamento del novembre-dicembre 1943 - per misurarsi con la società italiana, impegnandosi a cercare pragmaticamente nelle sue pieghe ogni forma possibile di sostegno o accettazione.

Ma per arrivare a definire le linee di contatto, e di possibile interazione, tra quelle pieghe e i corpi amministrativi della Rsi, non va dimenticato che esse sono principalmente il frutto dato dall'incrociarsi tra modelli innescati dal regime e frammentazioni e disgregazioni direttamente connesse agli eventi bellici. Da qui discende allora la necessità di rivolgersi anche a un altro fondamentale percorso interpretativo, apparentemente meno interno alla Rsi. Guerra, Resistenza, occupazione tedesca e debolezze di Salò, con la loro più o meno intensa presenza, si incaricarono di ridefinire gli spazi, accentuarono l'esistente o generarono rinnovati intrecci con quelle specificità e autonomie territoriali da sempre elemento chiave del nesso centro-periferia per tutta la nostra storia nazionale, e nodo cru-

Il dibattito sulla zona grigia ha in effetti riguardato essenzialmente la sua collocazione, la sua natura e il suo peso sull'Italia del dopoguerra. Luigi Ganapini l'ha indicata come «un'area sociale e culturale non facilmente definibile, in cui allignano motivazioni contraddittorie, ma questo non significa che non sia possibile [...] sciogliere - sia pure solo in parte - gli interrogativi concernenti la sua collocazione ideale» [Ganapini 1999, 253]. Renzo De Felice la fa coincidere con l'attesismo «che caratterizzò l'atteggiamento di larghissimi settori della popolazione rispetto all'occupazione tedesca, alla Rsi e alla resistenza» [De Felice 1997, 185]. E quindi, in altre parole, con l'area della passività, della non scelta, dell'estraneità alle sorti del conflitto, dove si sarebbe raccolta la maggioranza della popolazione, attenta principalmente alla sopravvivenza. Per Claudio Pavone essa «ha finito con l'indicare non solo tutti coloro che non vollero essere né vittime né persecutori, ma altresi quelli che non vollero essere ribelli all'oppressione e alla persecuzione. Ampiezza e caratteri di questa zona variano da paese a paese. Entro la comune cornice data dalla guerra e dall'occupazione, le zone grigie [...] traggono la loro particolare fisionomia da fattori di lungo periodo» [Pavone 1998, 6-7]. 
ciale della storia del fascismo per tutta la sua esistenza. Un percorso di lettura, tuttavia, di uso consolidato soprattutto per quanto riguarda l'analisi del regime, delle sue strutture e del suo consenso, ma in generale ritenuto questione assai marginale davanti alle vicende della Rsi e alle sue conclamate impotenze.

\section{Una proposta interpretativa}

Sono proprio questi i temi che si è inteso mettere in discussione in questo dossier, facendo seguito e sviluppando un filo interpretativo che è già stato al centro del convegno tenutosi nel settembre scorso a Ferrara ${ }^{4}$. Per la verità, l'opportunità di sondaggi in direzione delle strutture amministrative saloine, del loro funzionamento e della loro effettiva ricaduta (come canale aperto verso la società) era già stata richiamata da un altro convegno, tenutosi proprio a Salò una quindicina di anni fa. In quella occasione erano stati sottolineati i possibili limiti nelle fonti, e perciò anche interpretativi, di un simile approccio; ma anche le acquisizioni recenti e le potenzialità di un versante di ricerca su settori dello stato e delle organizzazioni di massa fasciste che, secondo il principale curatore dell'evento, in ambito amministrativo-gestionale avevano rappresentato un centro comunque dotato di una sovranità reale e continuativa $\mathrm{a}^{5}$.

Tuttavia, rispetto a questa visione, quello che si propone in questa sede è un punto di partenza completamente rovesciato. Serio può divenire, altrimenti, il rischio di dare eccessiva rilevanza a ciò che determinati segmenti del gruppo dirigente nazionale fascista, comunque dotati di poteri modesti, sostanzialmente dicevano di loro stessi. Più realistico sembra invece muovere dalla debolezza del centro, come elemento indiscutibile di una repubblica il cui governo risulta anche fisicamente polverizzato intorno al lago di Garda [Borghi 2008]. Certo, altrettanto indiscutibile è la febbrile attività amministrativa che caratterizza quel governo. Oltre alla mole considerevole di disposizioni e circolari emanate, ce

\footnotetext{
I molti territori della Repubblica fascista. Amministrazione e società nella RSI, Ferrara, Istituto di storia contemporanea e Dipartimento di scienze umane dell'Università degli studi, 27-28 settembre 2017.

Le fonti per la storia della RSI, Salò, Centro studi e documentazione sul periodo della Repubblica sociale italiana, 29 novembre 2003. Nel suo saggio incluso negli atti del convegno, Aldo Ricci precisa di riferirsi a quella vastissima zona grigia «che comprende quei maggioritari settori della società e degli apparati dello stato e delle amministrazioni locali che non aderivano per esplicita e cosciente scelta politica alla Rsi, ma condividevano comunque le necessità proprie di una vita associata di continuare a produrre beni di consumo, ma anche sentenze, istruzione, sanità, pensioni e così via» [Ricci 2005, 72]. Ovviamente, che fosse quel tipo di stato a garantire quelle necessità ben difficilmente poteva essere considerato ininfluente. In materia di precedenti relativi alla ricostruzione amministrativa dall'interno del centro si veda soprattutto il lavoro di Marco Borghi [2001].
} 
lo certifica la cartografia. Essa ci mostra infatti l'incessante riorganizzazione di enti e istituzioni; la continua mobilità della loro geografia, che se in taluni casi è evidentemente ricollegabile ai movimenti del fronte - nitido è ad esempio il picco registrato nell'estate 1944 -, in altri sembra procedere in autonomia, seguendo forse il progressivo conformarsi di un progetto di addensamento in area lombarda, di rafforzamento amministrativo e di qualificazione territoriale.

Di fatto, complici l'invadenza tedesca, le distruzioni belliche, la lotta partigiana e le pessime comunicazioni, la Rsi accentua rapidamente la sua frammentazione territoriale interna; assume cioè fin dal principio l'aspetto di un mosaico di aree solo relativamente collegate, piuttosto che di una compagine nazionale unitaria [Palla 1992, 236]. Qui tutti quei poteri, che pure torneranno ad essere nazionali alla fine della guerra ma che sono comunque all'opera, tendono ad apparire in una certa misura disaggregati e disarticolati. Al tempo stesso - e diverse indicazioni erano già venute, negli stessi anni Novanta, dalle ricerche sulle Città in guerra promosse dalla rete degli Istituti di storia della Resistenza ${ }^{6}$-, molti dei poteri locali conoscono di necessità un'amplificazione senza precedenti delle proprie funzioni e dei propri ambiti d'intervento.

Con l'avvertenza di mantenere comunque un quadro generale in cui calare il locale, partire dal centro sembra allora il piano meno adatto per verificare le reali modalità di governo e le ricadute delle strategie di consenso/accettazione messe in atto dalla Repubblica alla ricerca della necessaria stabilità. Di certo essa non ha il tempo né la forza di realizzare qualcosa di veramente nuovo e di effettivamente praticabile. Per dare sostanza al suo potere, la Rsi non può che cercare di aderire a singole realtà che, col procedere del conflitto, avevano visto rafforzarsi o incrinarsi specifiche strutture e continuità di fondo; deve misurarsi, nei suoi molti territori, con le ricadute dei modelli e delle strutture di modernizzazione e nazionalizzazione utilizzati dal regime e adeguati dalle diverse realtà nelle contingenze belliche; porsi con essi possibilmente in sintonia. È questa la sua unica concreta possibilità di governo. La sua consistenza politica e sociale appare perciò molto più legata alla somma di questi più o meno riusciti adeguamenti particolari (dove può trovare una collocazione un'idea anche funzionale della violenza), piuttosto che alla dubbia praticabilità delle grandi parole d'ordine continuamente sbandierate dal centro per tutti quei rapidi venti mesi. 


\section{Uomini, apparati e interessi}

Oltre al mantenimento di un ordine sempre precario, elementi cruciali dell'attività della Repubblica sono i servizi di assistenza e protezione, la mobilitazione e la gestione delle risorse umane e produttive. Su questo si fonda un'ampia fetta di quella sempre dubbia credibilità di cui il nuovo stato fascista ha bisogno davanti ai tedeschi riluttanti, e a tutti quei gruppi in qualche modo disposti a riconoscergli un ruolo.

In tutte queste direzioni giocano parti fondamentali istituzioni ed enti vari, in gran parte rapportabili direttamente all'impianto dello stato amministrativo e sindacalcorporativo degli anni Trenta, e tutti capillarmente distribuiti sul territorio nazionale. Tuttavia, da tempo gli studi di storia amministrativa hanno reinterpretato questo passaggio non più come un'azione a senso unico del centro, ma piuttosto come una «continua interrelazione negoziale» con le periferie [Baldissara 1998, 9]; e le più recenti rifioriture di studi sui fascismi locali hanno decisamente posto al centro della propria attenzione l'articolazione e la complessità delle traduzioni periferiche a cui sono soggette le politiche mussoliniane volte a subordinare e gerarchizzare le province [Gallerano 1991; Camurri, Cavazza e Palla 2010; Corner e Galimi 2014].

Accogliendo la scarsa linearità di questo rapporto Paul Corner, in un suo recente lavoro incentrato soprattutto sul partito e sul consenso negli anni Trenta, ha introdotto un interessante cambio di prospettiva, scegliendo di evitare il consueto "partire da Roma", «per estendere lo sguardo a come la periferia reagì agli impulsi provenienti dal centro»; e questo nella convinzione che «è soprattutto in questo ambito, quello del modo in cui le periferie reagirono alle iniziative dello stato centrale, che manchiamo ancora di conoscenze adeguate» [Corner 2015, 18].

Qualcosa di non troppo diverso vale senza dubbio anche per la costruzione di molti dei nuovi apparati dello stato amministrativo, pezzi non secondari della ricerca del consenso all'interno della contraddittoria modernizzazione intrapresa dal regime in quegli stessi anni Trenta. Pier Paolo D'Attorre ci ha avvertito che qui il prefetto «molto controlla e poco dirige; di fatto a lui molto sfugge e altrettanto è delegato ad altri» [D'Attorre 1991, 408]. Questo vale tanto per le reti previdenziali e assistenziali, quanto per la gestione burocratizzata delle risorse che viene progressivamente spostata nelle maglie delle commissioni miste, dei comitati orizzontali e verticali, degli organismi finanziari, consortili che caratterizzano la creazione degli apparati sindacal-corporativi, voluti nello sforzo totalitario e ormai bellicistico, in un «processo di statalizzazione dell'attività dei privati e del mercato» [Melis 1996, 368]. Sono tutti canali a doppio senso, ampiamente dira- 
mati, che gli interessi privati riempiono, in cui possono fondersi vecchi e nuovi gruppi della classe dirigente fascista; in cui le trasformazioni imposte dalla guerra non di rado rafforzano, e senza troppi strappi, i margini delle autonomie periferiche, nonostante il centro tenti, per tutto il corso del conflitto, di modellarne e rimodellarne sempre più freneticamente i ruoli.

Al di là di leve militari, polizie speciali e tribunali straordinari che pure sono parte integrante del nuovo stato fascista, sono questi gli apparati e i poteri (occupati da uomini di cui ancora molto poco si sa') chiamati ad essere i principali interlocutori delle molteplici frange della zona grigia, a fondersi con essa, ad avere perciò una parte almeno altrettanto significativa per Salò. A questi andarono a collegarsi strutture chiave della Rsi e dell'alleato-occupante, e a loro continuarono a essere chiesti contributi fondamentali, legati alla produzione, alla distribuzione e alla protezione del lavoro e delle risorse. Illuminanti possono essere, in questo senso, tanto le vicende del sindacato dei lavoratori dell'agricoltura e dell'ispettorato del lavoro, quanto quelle degli organismi deputati a giocare l'ambigua partita della discriminazione e della gestione dei beni ebraici. L'esplicita fascistizzazione di alcuni di questi livelli pare essere lasciata appositamente indeterminata dalla Rsi, ma è probabile che, nella sostanza, essi finiscano per poggiare su marcate continuità con gli anni Trenta.

Dunque, in movimento tra le società locali e la sala delle udienze di Mussolini a Gargnano, mescolata a ministri, generali e dirigenti del partito, è insistentemente riconoscibile una lunga sequenza di esponenti a vario titolo e a vario grado dei poteri qui in discussione. Essa va dai podestà ai rappresentanti della giustizia ordinaria, ai tecnici legati a ispettorati, commissariati, consorzi e sezioni produttive varie; dai giornalisti ai dirigenti di vecchie (e formalmente soppresse) o nuove confederazioni e unioni sindacali; soprattutto ai nuovi prefetti/capi provincia che, verso la fine del 1943. Buffarini è riuscito finalmente ad insediare in tutto il territorio repubblicano.

Sono proprio questi ultimi il nostro primo livello di riferimento, quelli che compongono, secondo tradizione, i vertici delle gerarchie amministrative (e ora, almeno nelle intenzioni, anche di quelle politiche) periferiche. Oggetto di almeno quattro grandi movimenti di sedi (autunno 1943, primavera e poi estate 1944, inizio 1945), e in generale di frequenti spostamenti e rimozioni, essi restano comunque i principali raccordi con il centro, i canali delle sue crescenti pulsioni 
totalitarie e filonaziste; ma sono anche coloro che si sforzano di governare le piccole repubbliche in cui si va frazionando il territorio saloino, impegnati a cercare, a dispetto dei continui interventi ministeriali e delle ripetute licenze germaniche, alleanze variabili e a breve raggio, nello sforzo di potersi comunque garantire risorse e stabilità'. In realtà, finora non molto si sa né si è aggiunto a quanto già si sapeva su costoro; e sul loro inquadramento interpretativo pesano tuttora generalizzazioni e decontestualizzazioni in parte fuorvianti. Il moderato Piero Parini e l'intransigente e fanatico Enrico Vezzalini - classici esempi della più corrente caratterizzazione riservata agli esponenti della Rsi - a Milano e a Ferrara hanno in comune successi politici e amministrativi difficili da comprendere se mantenuti all'interno di ripartizioni troppo secche e, soprattutto, se portati fuori dai contesti, comunque molto diversi, in cui si trovarono ad agire [Ganapini 1988; Parisini 2005].

Sono anche questi percorsi a cui è sembrato opportuno cominciare a mettere mano, in quanto lasciano intendere profili e intrecci anche diversi da quelli considerati ormai consolidati. E non va poi dimenticato che fame, bombe e sfollamenti (ma anche organizzazione del tempo libero in città come in campagna) coinvolgono direttamente anche il partito. La sua presenza, talvolta accattivante (si vedano ad esempio i "colloqui del giovedi" col federale promossi sulla stampa fiorentina), spesso ingombrante, impone complesse dialettiche che attraversano tutti i corpi di Salò, e include l'obbligo di indagarne almeno l'accresciuto ruolo di organizzatore della vita materiale degli italiani.

\section{Quali territori?}

Muovendo da tali presupposti, è allora dalla ricostruzione di questi specifici intrecci di poteri, apparati, reti di interessi e uomini all'interno delle specifiche realtà che occorre partire per caratterizzare davvero consistenza e ricadute dell'azione amministrativa della Repubblica fascista. Ma quali sono queste realtà, quale la loro dimensione territoriale? Il punto di partenza di ogni considerazione in questo senso non può ovviamente non poggiare sugli studi locali che, a varie riprese, sono stati prodotti nell'arco di questi ultimi decenni, a testimonianza di un soggetto storico che non ha mai smesso di esercitare un considerevole appeal. 
Questi lavori costituiscono un insieme sufficientemente articolato da coprire la maggior parte del territorio su cui la Rsi esercitò un dominio abbastanza duraturo. La scala di riferimento privilegiata è, naturalmente, quella provinciale, e questa rimane un primo fondamentale passaggio per la valorizzazione di un'interessante varietà di fonti, per le ovvie praticità di riferimenti documentari, comparativi e interpretativi. Tale scala si è dunque mantenuta anche nell'articolazione della maggior parte dei saggi che compongono questo dossier, diversi dei quali sono stati affidati proprio agli autori di alcuni dei più recenti tra quei lavori, e tra i più sensibili ai temi qui in questione.

Tuttavia, vale la pena di sviluppare a questo proposito qualche riflessione aggiuntiva, rispetto a una dimensione che può essere utilmente variata sulla base di riferimenti ancora più adeguati a cogliere l'effettiva incidenza dei processi qui in esame. Se è apparso quantomeno utile collegarsi alle emanazioni dello stato amministrativo, alla pervasività delle sue maglie così come vengono messe a punto negli anni del regime, è proprio sulla scala indirettamente suggerita da questi riferimenti che, allo stesso modo, appare necessario allargare gli orizzonti delle nostre ricerche.

I gruppi di pressione chiamati all'azione da questi intrecci sono in realtà più territoriali che provinciali. Essi ci richiamano infatti a una maggiore corrispondenza con i profili più flessibili ed economicamente specializzati delle diverse Italie agricole e industriali. Territori perciò che possono essere più ampi o più intrecciati dei consueti confini amministrativi o di quelli (che pure rimangono fondamentali) tra città e campagne; aree che, senza dimenticare ineludibili specificità locali, hanno elaborato sul lungo e medio periodo caratteristiche sostanzialmente accomunabili dal punto di vista dei tessuti sociali e delle culture politiche. E di cui certamente occorre verificare persistenze, comunanze o ridefinizioni alla luce del multiforme intreccio e della varia consistenza delle variabili direttamente innescate dalla guerra.

Si può allora cominciare a considerare alcuni primi quadri esemplificativi tanto nell'area, per tanti versi già ampiamente scandagliata, dei grandi centri del cosiddetto triangolo industriale; quanto in quella, fisicamente non molto distante e pure ad alta densità operaia, che si estende tra il Bresciano e il Vicentino. Territori dalle caratteristiche economiche a prima vista certo non molto dissimili, e pure con prospettive ed esiti piuttosto diversi, su cui non ha mancato di richiamare l'attenzione, tra gli altri, Santo Peli [1999] nei suoi studi sulla «Resistenza difficile». Allo stesso modo pare lecito delineare, intorno all'asse Pisa-Firenze, un'area agricolo-manifatturiera con una variegata presenza di ceto intermedio, in cui racchiudere tutta una parte della Toscana settentrionale [Preti 1986]; mentre indiscu- 
tibilmente netto si presenta il profilo di un'intera fascia di pianura che, a cavallo del Po, va dalle basse Lodigiana e Bresciana, dal Cremonese fino al Polesine e a tutta la bonifica ferrarese. Qui, le aziende capitalistiche avevano fortemente modellato strutture agrarie e sociali, come ci ha ben mostrato una ricca e importante stagione di studi locali; qui, per tornare al nostro argomento, tanto il regime quanto la Repubblica sociale ottennero a lungo, con poche eccezioni, esemplari indici produttivi, consegne agli ammassi piuttosto puntuali, e continuarono a collocare importanti quote di debito pubblico.

È in quadri come questi che pare utile cominciare a collocare e a verificare quegli intrecci di cui si parlava più sopra. È da qui che, verosimilmente, possono prendere una certa consistenza le molte ambiguità e le indubbie fragilità della struttura amministrativa della Rsi, suggerendoci possibili ulteriori periodizzazioni e, eventualmente, più articolate puntualizzazioni già sbilanciate sul dopo. Perché, se quanto detto sin qua ha qualche fondamento, la continuità, ovunque e fino a quando fu possibile, potrebbe essere il dato più incisivo della Repubblica fascista, in vista tanto del governo delle emergenze belliche, quanto di un qualche dopoguerra. Almeno sino alla disperata militarizzazione finale, o piuttosto finchè il fronte non diventi così vicino da sommergere inevitabilmente ogni possibile equilibrio.

\section{Bibliografia}

Baldissara L. 1998, Tecnica e politica dell'amministrazione. Saggio sulle culture amministrative e di governo municipale fra anni Trenta e Cinquanta, Bologna: il Mulino

Borghi M. 2001, Tra fascio littorio e senso dello stato. Funzionari, apparati, ministeri nella Repubblica sociale italiana (1943-1945), Padova: Cleup

Borghi M. 2008, Un arcipelago di "non luoghi" per il fascismo estremo, in Isnenghi M. e Albanese G. (eds.) 2008, Gli Italiani in guerra, vol.IV, t.II, Il Ventennio fascista: la Seconda guerra mondiale, Torino: Utet

Camurri R., Cavazza S. e Palla M. (eds.) 2010, Fascismi locali, "Ricerche di storia politica", 3

Corner P. 2015, Italia fascista. Politica e opinione popolare sotto la dittatura, Roma: Carocci

Corner P. e Galimi V. (eds.) 2014, Il fascismo in provincia. Articolazione e gestione del potere tra centro e periferia, Roma: Viella

Dalla Casa B. e Preti A. (eds.) 1995, Bologna in guerra 1940-1945, Milano: FrancoAngeli

D'Attorre P.P. 1991, Aspetti economici e territoriali del rapporto centro/periferia, "Italia contemporanea", 184 
De Felice R. 1997, Mussolini l'alleato 1940-1945, vol.II, La guerra civile 1943-1945, Torino: Einaudi

Della Valle C. 1978, Repubblica sociale italiana, in Levi F., Levra U. e Tranfaglia N. (eds.) 1978, Storia d'Italia, vol. III, Firenze: La Nuova Italia

Gallerano N. 1991, Le ricerche locali sul fascismo, "Italia contemporanea", 184

Gagliani D. 1999, Brigate nere. Mussolini e la militarizzazione del Partito fascista repubblicano, Torino: Bollati Boringhieri

Ganapini L. 1988, Una città, la guerra; lotte di classe, ideologia e forze politiche a Milano 1939-1951, Milano: FrancoAngeli

Ganapini L. 1999, La repubblica delle camicie nere. I combattenti, i politici, gli amministratori, i socializzatori, Milano: Garzanti

Ganapini L. 2000, La Rsi e l'ultimo fascismo. Una rilettura critica della storiografia, "L'impegno", 3

Klinkhammer L. 1993, L'occupazione tedesca in Italia 1943-1945, Torino: Bollati Boringhieri

Legnani M. 1986, Potere, società ed economia nel territorio della RSI, in P.P. Poggio (ed.) 1986, La Repubblica sociale italiana, “Annali”, 2, Brescia: Fondazione L. Micheletti

Melis G. 1996, Storia dell'amministrazione italiana 1861-1993, Bologna: il Mulino

Osti Guerrazzi A. 2012, Storia della Repubblica sociale italiana, Roma: Carocci

Palla M. 1992, Amministrazione periferica e fonti locali sul collaborazionismo in Italia durante la RSI, in L. Cajani e B. Mantelli (eds.) 1992, Una certa Europa. Il collaborazionismo 1939-1945, “Annali”, 6, Brescia: Fondazione L. Micheletti

Parisini R. 2005, Dal regime corporativo alla repubblica sociale. Agricoltura e fascismo a Ferrara, 1928-1945, Ferrara: Corbo

Pavone C. 1991, Una guerra civile. Saggio storico sulla moralità nella Resistenza, Torino: Bollati Boringhieri

Pavone C. 1995, Alle origini della repubblica. Scritti su fascismo, antifascismo e continuità dello Stato, Torino: Bollati Boringhieri

Pavone C. 1998, Caratteri ed eredità della zona grigia, "Passato e presente", 43

Peli S. 1999, La Resistenza difficile, Milano: FrancoAngeli

Poggio P.P. (ed.) 1986, La Repubblica sociale italiana, “Annali”, 2, Brescia, Fondazione L. Micheletti

Poggio P.P. e Sciola G. 1982, Le fonti della Repubblica sociale italiana per lo studio della questione contadina durante la seconda guerra mondiale, in Istituto A. Cervi (ed.) 1982, Agricoltura e contadini in Lombardia tra guerra e resistenza, "Annali", 4

Preti D. 1986, Tra crisi e dirigismo: l'economia toscana nel periodo fascista, in Mori G. (ed.) 1986, Storia d'Italia. Le Regioni dall'Unità ad oggi. La Toscana, Torino: Einaudi 
Ricci A.G. 2005, Governo e amministrazione nella Rsi: fonti istituzionali e prospettive di ricerca, in Id. (ed.) 2005, Le fonti per la storia della RSI, Venezia: Marsilio

Rovatti T. 2011, Leoni vegetariani. La violenza fascista durante la Rsi, Bologna: Clueb

Rovatti T. 2014, Linee di ricerca sulla Repubblica sociale italiana, "Studi storici”, 1

Rusconi G. E. 1995, Resistenza e postfascismo, Bologna: il Mulino

Scoppola P. 1995, 25 aprile. Liberazione, Torino: Einaudi

Ventrone A. 2017, Il fascismo non è una causa perduta. Ricordi e rimozioni nei vinti della Repubblica sociale italiana, "Meridiana", 88

Vinci A.M. 1993, Trieste in guerra. Gli anni 1938-1943, Trieste: Sciarada

\section{Risorse}

Programma del Convegno I molti territori della Repubblica fascista. Amministrazione e società nella RSI, Ferrara, 27-28 settembre 2017

https:/e-review.it/sites/default/images/articles/media/191/programma convegno.pdf 